\title{
COVID-19 y ansiedad en oftalmólogos
}

\section{COVID-19 and anxiety in ophtalmologists}

\author{
José R. Mier-Bolio 1*, José M. Arroyo-González¹, Elizabeth Baques-Guillén ${ }^{1}$, José F. Valdez-Lopez', \\ Álvaro J. Torre-García², Olivia E. Rodríguez-Rodríguez ${ }^{3}$ y Gerardo Rivera-Arroyo ${ }^{1}$
}

'Servicio de Retina y Vítreo, Hospital Central Militar; ${ }^{2}$ Servicio de Cardiología Intervencionista, Instituto Nacional de Cardiología Ignacio Chávez; ${ }^{3}$ Servicio de Cardiología, Escuela Militar de Graduados de Sanidad, Universidad de Ejército y Fuerza Aérea. Ciudad de México, México

\section{Resumen}

Objetivo: Determinar la prevalencia de ansiedad en oftalmólogos durante la pandemia COVID-19 (enfermedad por coronavirus 2019), expuestos al síndrome respiratorio agudo severo por coronavirus tipo 2 (SARS-CoV-2) laborando en el triaje respiratorio y en la consulta externa de oftalmología en el Hospital Central Militar de la Ciudad de México. Material y métodos: Estudio observacional, transversal, descriptivo y prospectivo, en el cual se aplicó una encuesta descriptiva vía Internet en la que se valoraron dos escalas de ansiedad (escala de Hamilton y escala del trastorno de ansiedad generalizada [GAD- ${ }^{7}$ ). Resultados: Se estudiaron 31 personas, 17 (54.84\%) mujeres y 14 (45.16\%) hombres. La edad mínima fue de 25 años, la máxima, de 49 años y el promedio, de 34.5 años. La encuesta estaba compuesta por 24 preguntas, de las cuales, 21 se obtuvieron al unir las escalas de ansiedad (Hamilton + GAD-7), las tres restantes de interés epidemiológico. Al valorar ambas escalas por separado, obtuvimos una puntuación promedio de 5.16 para la escala GAD-7 y de 8.45 para la de Hamilton, lo que refleja una ansiedad mínima grupal. Usando la escala GAD-7 se observó una prevalencia de ansiedad del $48 \%$ (15 médicos). De los casos diagnosticados, 9 (60\%) cumplieron un puntaje catalogado como ansiedad leve, 5 (53\%), como moderada y 1 (7\%), como severa. Con la escala de Hamilton, la prevalencia fue del 83\% (26 médicos). De los casos diagnosticados, 23 (88\%) con ansiedad mínima, 2 (8\%) con ansiedad mínima-moderada y 1 (4\%) con ansiedad severa. Conclusión: La prevalencia de ansiedad en oftalmólogos, usando la escala GAD-7 y la escala de Hamilton, fue del 48 y $83 \%$, respectivamente, la mayoría de intensidad leve.

Palabras clave: Ansiedad. Oftalmólogos. COVID-19. SARS-CoV-2. Pandemia.

\section{Abstract}

Objective: To determine the prevalence of anxiety in ophthalmologists during the COVID-19 (coronavirus disease 2019) pandemic, exposed to severe acute respiratory syndrome by coronavirus type 2 (SARS-CoV-2) working in respiratory triage and outpatient ophthalmology consultation at the Hospital Central Militar in Mexico City. Material and methods: Observational, cross-sectional, descriptive and prospective study, in which a descriptive survey was applied via internet evaluating two anxiety scales (Hamilton scale and generalized anxiety disorder [GAD-7) scale). Results: Thirty-one subjects responded, 17 (54.84\%) female and 14 (45.16\%) male. Minimum age was 25 years and maximum age was 49 years, with an average age of 34.5 years. The survey consisted of 24 questions, of which 21 were obtained from both anxiety scales (Hamilton + GAD-7)

\section{Correspondencia:}

*José R. Mier-Bolio

Periférico Blvrd Manuel Ávila Camacho, s/n

Del. Miguel Hidalgo

Fecha de recepción: 13-06-2020

Fecha de aceptación: 22-07-2020

C.P. 11200 Ciudad de México, México

E-mail: josemierb @gmail.com (http://creativecommons.org/licenses/by-nc-nd/4.0/). DOI: 10.24875/RMO.M20000130
Disponible en internet: 17-09-2020 Rev Mex Oftalmol. 2020;94(6):247-251

www.rmo.com.mx 
and the remaining three were of epidemiological interest. By rating both scales separately, we observed an average score of 5.16 for the GAD-7 scale and of 8.45 for the Hamilton scale, reflecting a minimal group anxiety. Using the GAD-7 scale, we observed an anxiety prevalence of $48 \%$ (15 physicians). Of the diagnosed cases, 9 (60\%) met a score listed as mild anxiety, $5(53 \%)$ as moderate and $1(7 \%)$ as severe. On the Hamilton scale, the prevalence was of $83 \%$ (26 physicians). Of the diagnosed cases, 23 (88\%) had minimal anxiety, 2 (8\%) had minimal-moderate anxiety and 1 (4\%) had severe anxiety. Conclusion: The prevalence of anxiety in ophthalmologists, using the GAD-7 and Hamilton scales was of $48 \%$ and $83 \%$, respectively, and most cases were of mild intensity.

Key words: Anxiety. Ophthalmologists. COVID-19. SARS-CoV-2. Pandemic.

\section{Introducción}

La Guía de Práctica Clínica Mexicana sobre el «Diagnóstico y Tratamiento de los Trastornos de Ansiedad en el Adulto" define la ansiedad como un estado emocional displacentero que se acompaña de cambios somáticos y psíquicos, que puede presentarse como una reacción adaptativa o como un síntoma o síndrome que acompaña a diversos padecimientos médicos y psiquiátricos. Se considera que la ansiedad es patológica por su presentación irracional, ya sea porque el estímulo está ausente, la intensidad es excesiva con relación al estímulo o la duración es injustificadamente prolongada y la recurrencia inmotivada, generando un grado evidente de disfuncionalidad en la persona'.

La pandemia originada por el brote del nuevo virus, síndrome respiratorio agudo severo por coronavirus tipo 2 (SARS-CoV-2), desde sus inicios se ha encontrado fuertemente ligado con el gremio de la oftalmología ${ }^{2-3}$. Uno de los pioneros en descubrir el brote fue el Dr. Li Wenliang, oftalmólogo de profesión y originario de China, laborando en el Hospital Central Wuhan en la provincia de Hubei, China ${ }^{2-5}$. El Dr. Li Wenliang se infectó de un paciente asintomático con diagnóstico de glaucoma por cierre agudo angular. El día 10 de enero del 2020 el Dr. Li manifestó síntomas del nuevo virus y falleció el 7 de febrero del $2020^{2}$.

En México no tenemos lo necesario para combatir esta pandemia. Es bien sabido que no se cuenta con el personal de salud suficiente, la capacidad hospitalaria y los insumos necesarios, por lo que el personal de salud es de las poblaciones con mayor riesgo de desarrollar trastornos de ansiedad. Algunos de los factores que pueden desencadenar la ansiedad en el personal de salud son: las largas jornadas laborales, el riesgo de adquirir la infección, no contar con el equipamiento completo de protección, la soledad, la fatiga física y emocional, así como el distanciamiento de los seres queridos ${ }^{6-9}$.

Se han diseñado múltiples escalas para medir la severidad de los trastornos de ansiedad. Dos de las escalas más utilizadas a nivel mundial y previamente aprobadas en la población mexicana son: la escala de Hamilton y la escala para el trastorno de ansiedad generalizada (GAD-7). Ambas se basan en una serie de preguntas sobre eventos que pudiesen estar desencadenando ansiedad, y, de acuerdo con las respuestas, se realiza una sumatoria de los reactivos, a mayor ansiedad mayor la sumatoria de los reactivos ${ }^{10-11}$.

El propósito del siguiente estudio es conocer la prevalencia de ansiedad en un grupo de oftalmólogos laborando durante la pandemia COVID-19 en un hospital de tercer nivel.

\section{Material y métodos}

Se realizó una encuesta descriptiva, con un diseño metodológico observacional, transversal, descriptivo y prospectivo. El objetivo primario fue determinar la prevalencia de ansiedad en los oftalmólogos a cargo de la atención de pacientes durante la pandemia COVID-19.

El estudio fue realizado al personal de oftalmología de todas las subespecialidades del Hospital Central Militar, en la Ciudad de México, durante el mes de mayo del 2020. Todos los participantes firmaron previamente el consentimiento informado.

Nuestros criterios de inclusión fueron: género indistinto, no presentar enfermedades crónico-degenerativas o enfermedad psiquiátrica diagnosticada previamente 0 bajo tratamiento y formar parte del personal del servicio de oftalmología atendiendo pacientes durante la pandemia COVID-19. Esto incluyó: residentes, becarios de alta especialidad y médicos adscritos de las distintas subespecialiades de oftalmología.

Se realizó un cuestionario, el cual constó de 24 preguntas. La encuesta fue aplicada vía Internet utilizando la plataforma SurveyMonkey ${ }^{\circledR}$ durante el mes de mayo del 2020. Se midieron los niveles de ansiedad utilizando las escalas de ansiedad, de Hamilton y la GAD-7, ambas previamente validadas en población mexicana. 
Tabla 1. Resultados por sexo de la escala del trastorno de ansiedad generalizada (GAD-7)

\begin{tabular}{|l|c|c|c|c|}
\hline Sexo & Puntuación mínima & Puntuación máxima & Puntuación promedio & Número de médicos con diagnóstico de ansiedad \\
\hline Femenino & 0 & 17 & 5.5 & $8(53 \%)$ \\
\hline Masculino & 0 & 10 & 4.6 & $7(47 \%)$
\end{tabular}

Las escalas se interpretan con un valor numérico ascendente. La escala de Hamilton consta de 14 preguntas, con valores que van del 0 a 4; la severidad de la ansiedad es interpretada con el resultado final por la suma de los reactivos, presentando una ansiedad mínima con un valor menor o igual a 17 , mínima a moderada con valores de 18 a 24 , moderada con valores de 25 a 30 y severa con un valor mayor a 30.

La escala de GAD-7, que valora el estado de ansiedad en las últimas 2 semanas, está conformada por 7 preguntas, con valores de 0 a 3 , e igualmente, la severidad de la ansiedad es interpretada por la sumatoria de los reactivos, reportando una ansiedad mínima con valores entre 5 y 9 , moderada con valores entre 10 y 14 y severa con 15 o más reactivos.

\section{Resultados}

La totalidad de la población estudiada completó la encuesta al $100 \%$, con un tiempo promedio de 3 minutos.

El estudio incluyó 31 personas, 17 (54.84\%) mujeres y 14 (45.16\%) hombres. La edad mínima fue de 25 años, la máxima, de 49 años y la edad promedio, de 34.5 años (Anexo 1).

La edad promedio dividida por sexos fue de 33.47 años para el sexo femenino y de 35.85 para el sexo masculino. La variable de interés primario fue determinar la ansiedad en oftalmólogos laborando en un hospital de tercer nivel durante la pandemia COVID-19.

Realizamos una encuesta utilizando la plataforma SurveyMonkey ${ }^{\circledR}$ vía Internet, compuesta en su totalidad por 24 preguntas, de las cuales, 21 fueron obtenidas al unir ambas escalas de ansiedad (Hamilton + GAD-7), donde se obtuvieron una totalidad de 77 reactivos. Las 3 preguntas restantes no influyeron en la sumatoria de los reactivos, fueron de interés epidemiológico: edad, sexo e iniciales del nombre.

Usando la escala GAD-7 se observó una prevalencia de ansiedad del 48\% (15 personas) del total de la muestra, $9(60 \%)$ personas cumplieron un puntaje catalogado como ansiedad leve, 5 (33\%) personas como

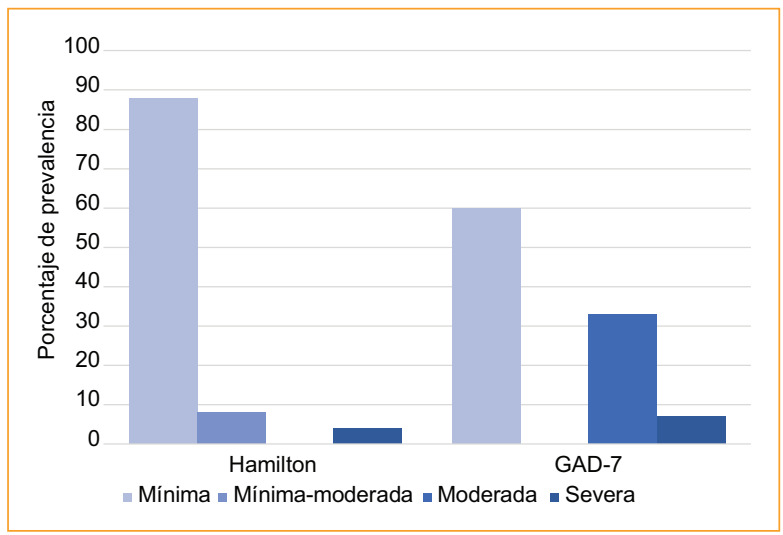

Figura 1. Prevalencia y severidad de ansiedad representada en porcentajes.

ansiedad moderada y $1(7 \%)$ persona como ansiedad severa (Fig. 1).

Detalladamente analizamos la escala GAD-7, donde obtuvimos un valor mínimo de 0 , máximo de 17 y promedio de 5.16, lo que refleja un desorden de ansiedad leve de manera grupal.

El análisis por sexo se representa en la tabla 1.

Utilizando la escala Hamilton se observó una prevalencia de ansiedad del $83 \%$ (26 personas) del total de la muestra, $23(88 \%)$ cumplieron un puntaje catalogado como ansiedad mínima, 2 (8\%) como mínima-moderada y 1 (4\%) como severa (Fig. 1).

Al realizar un análisis completo de la escala de Hamilton, los resultados que obtuvimos fueron los siguientes: la puntuación mínima fue de 0 , la máxima, de 43 y la promedio, de 8.45 , lo que refleja una severidad de ansiedad mínima de manera grupal.

Los resultados divididos por sexo se representan en la tabla 2.

\section{Discusión}

El presente estudio analizó, a través de una encuesta descriptiva, los niveles de ansiedad de médicos oftalmólogos. Se utilizaron dos de las escalas más difundidas a nivel mundial, la escala GAD-7 y la escala 
Rev Mex Oftalmol. 2020;94(6)

Tabla 2. Resultado por sexo de la escala de Hamilton

\begin{tabular}{|l|c|c|c|c|}
\hline Sexo & Puntuación mínima & Puntuación máxima & Puntuación promedio & Número de médicos con diagnóstico de ansiedad \\
\hline Femenino & 0 & 20 & 7.5 & $13(50 \%)$ \\
\hline Masculino & 0 & 43 & 9.5 & $13(50 \%)$
\end{tabular}

Hamilton, ambas previamente aprobadas en población mexicana. La población estudiada se conformó por 31 oftalmólogos, laborando durante la pandemia COVID19, expuestos al virus SARS-CoV-2 en la consulta externa por urgencias oftalmológicas y en la valoración del triaje respiratorio.

Se obtuvo un puntaje final por la escala GAD-7 de 5.16 puntos y por la escala de Hamilton de 8.45 puntos, ambos corresponden a un desorden de ansiedad de severidad leve de manera grupal.

Usando la escala GAD-7 se observó una prevalencia de ansiedad del 48\% (15 médicos). Del total de la muestra, $9(29 \%)$ cumplieron un puntaje catalogado como ansiedad leve, 5 (16\%) como moderada y 1 como severa. Con la escala Hamilton, la prevalencia fue del $83 \%$ (26 médicos), 23 personas (74\%) con ansiedad mínima, 2 como mínima-moderada, y una persona con ansiedad severa.

A diferencia, Cuiyan Wang, et al., en China, a través de una encuesta en línea generada para la población en general, un tercio de los 1,210 participantes reportaron un trastorno generalizado de ansiedad con severidad moderada a severa ${ }^{7}$.

Ravi Philip Rajkumar en una revisión sistematizada de la relación de la COVID-19 con la salud mental menciona que, dentro de todas las alteraciones de salud mental que pudiesen presentar las personas, el trastorno por ansiedad es el más común ${ }^{12}$.

La Secretaría de Salud de México estima que un $70 \%$ de la población mexicana se infecte por COVID19 , lo que ocasionaría el colapso hospitalario y la saturación de los hospitales debido a que no se cuenta con un sistema de salud para afrontar una pandemia de este nivel, por lo que se percibe un nivel de ansiedad en el personal de salud ${ }^{4}$.

Los niveles de ansiedad generados por la pandemia, específicamente al personal de salud, los afrontará de manera distinta por cada persona. Algunos de los factores de riesgo desencadenantes de ansiedad son: la edad, el género, la especialidad o capacitación del personal, enfermedades psiquiátricas previas o experiencias personales vividas durante la duración de la pandemia ${ }^{6-8}$.
Está claro que la pandemia por COVID-19 ha generado una respuesta por los servicios de psiquiatría y profesionales relacionados, en la cual los trastornos mentales son tomados a consideración especialmente en el personal de salud, de tal manera que resulta conveniente conocer la prevalencia de esta enfermedad en diferentes grupos de riesgo, para así distribuir los recursos humanos de manera eficiente ${ }^{12-13}$.

Hasta el momento es el primer estudio mexicano en documentar la prevalencia de ansiedad en oftalmólogos mexicanos laborando en un hospital de tercer nivel, durante la pandemia por COVID-19.

\section{Conclusión}

La prevalencia de ansiedad en oftalmólogos, usando la escala GAD-7 y Hamilton, fue del $48 \%$ y $83 \%$, respectivamente, la mayoría de intensidad leve.

\section{Financiamiento}

Los autores no recibieron patrocinio para llevar a cabo este artículo.

\section{Conflicto de intereses}

Los autores manifiestan no tener ningún conflicto de intereses.

\section{Responsabilidades éticas}

Protección de personas y animales. Los autores declaran que para esta investigación no se han realizado experimentos en seres humanos ni en animales.

Confidencialidad de los datos. Los autores declaran que han seguido los protocolos de su centro de trabajo sobre la publicación de datos de pacientes.

Derecho a la privacidad y consentimiento informado. Los autores han obtenido el consentimiento informado de los pacientes y/o sujetos referidos en el artículo. 


\section{Anexo}

Anexo 1. Resumen de resultados de la población estudiada.

\begin{tabular}{|c|c|c|c|c|}
\hline Médico oftalmólogo & Edad (años) & $\begin{array}{c}\text { Sexo } \\
F=\text { Femenino } \\
M=\text { Masculino }\end{array}$ & GAD-7 & Hamilton \\
\hline 1 & 29 & $\mathrm{M}$ & 3 & 7 \\
\hline 2 & 40 & $\mathrm{M}$ & 0 & 5 \\
\hline 3 & 31 & $F$ & 10 & 14 \\
\hline 4 & 48 & $\mathrm{M}$ & 7 & 11 \\
\hline 5 & 36 & $\mathrm{M}$ & 3 & 8 \\
\hline 6 & 34 & $\mathrm{M}$ & 6 & 9 \\
\hline 7 & 32 & $\mathrm{~F}$ & 7 & 3 \\
\hline 8 & 37 & $F$ & 4 & 8 \\
\hline 9 & 32 & $F$ & 2 & 7 \\
\hline 10 & 36 & $\mathrm{M}$ & 9 & 43 \\
\hline 11 & 42 & $F$ & 1 & 2 \\
\hline 12 & 42 & $F$ & 0 & 0 \\
\hline 13 & 43 & $F$ & 0 & 0 \\
\hline 14 & 28 & $F$ & 4 & 5 \\
\hline 15 & 36 & $F$ & 12 & 20 \\
\hline 16 & 28 & $\mathrm{M}$ & 0 & 1 \\
\hline 17 & 32 & $F$ & 1 & 7 \\
\hline 18 & 34 & $F$ & 1 & 0 \\
\hline 19 & 30 & $F$ & 11 & 2 \\
\hline 20 & 40 & $\mathrm{M}$ & 9 & 8 \\
\hline 21 & 25 & $\mathrm{~F}$ & 0 & 0 \\
\hline 22 & 28 & $\mathrm{M}$ & 7 & 8 \\
\hline 23 & 29 & $\mathrm{~F}$ & 7 & 9 \\
\hline 24 & 29 & $\mathrm{~F}$ & 6 & 16 \\
\hline 25 & 29 & $\mathrm{M}$ & 2 & 1 \\
\hline 26 & 30 & $\mathrm{M}$ & 0 & 0 \\
\hline 27 & 49 & $\mathrm{M}$ & 0 & 1 \\
\hline 28 & 32 & $\mathrm{~F}$ & 12 & 15 \\
\hline 29 & 30 & $\mathrm{M}$ & 9 & 17 \\
\hline 30 & 35 & $F$ & 17 & 20 \\
\hline 31 & 45 & $\mathrm{M}$ & 10 & 15 \\
\hline
\end{tabular}

GAD-7: escala del trastorno de ansiedad generalizada.

\section{Bibliografía}

1. CENETEC Trastornos de ansiedad. Guía de Referencia Rápida. Diagnóstico y tratamiento de los trastornos de ansiedad en el adulto. 2010 Disponible en: http://www.cenetec.salud.gob.mx/descargas/gpc/ CatalogoMaestro/392_IMSS_10_Ansiedad/GRR_IMSS_392_10.pdf

2. Parrish RK II, Stewart MW, Duncan Powers SL. Ophthalmologists Are More Than Eye Doctors-In Memoriam Li Wenliang. Am J Ophthalmol. 2020;213:A1-A2.

3. Wenliang L, Year LN. All eyes on Coronavirus - What do we need to know as ophthalmologists. Indian J Ophthalmol. 2020;68(4):549-53.

4. Emparan JPO, Garza-Leon M, García-Aguirre G, Azcárate-Coral T, Penniecook JA, Lansingh VC, et al. Recomendaciones para el manejo de pacientes que requieren atención oftalmológica durante la pandemia de SARS-CoV-2. Rev Mex Oftalmol. 2020;94(3):103-12.

5. Harapan H, Itoh N, Yufika A, Winardi W, Keam S, Te H, et al. Coronavirus disease 2019 (COVID-19): A literature review. J Infect Public Health. $2020 ; 13: 667-73$

6. Shanafelt T, Ripp J, Sinai M, Trockel M. Understanding and Addressing Sources of Anxiety Among Health Care Professionals During the COVID-19 Pandemic. JAMA. 2020;323(21):2133-4.

7. Wang C, Pan R, Wan X, Tan Y, Xu L, Ho CS, et al. Immediate psychological responses and associated factors during the initial stage of the 2019 coronavirus disease (COVID-19) epidemic among the general population in China. Int J Environ Res Public Health. 2020; 17(5):1729.

8. Tsamakis K, Rizos E, Manolis A, Chaidou S, Kympouropoulos S, Spartalis $\mathrm{E}$, et al. [Comment] COVID-19 pandemic and its impact on mental health of healthcare professionals. Exp Ther Med. 2020;19(6): 3451-3

9. Kang L, Li Y, Hu S, Chen M, Yang C, Yang BX, et al. The mental health of medical workers in Wuhan, China dealing with the 2019 novel coronavirus. The Lancet Psychiatry. 2020;7(3):e14.

10. Ruiz MA, Zamorano E, García-Campayo J, Pardo A, Freire O, Rejas J. Validity of the GAD-7 scale as an outcome measure of disability in patients with generalized anxiety disorders in primary care. J Affect Disord. 2011;128(3):277-86.

11. Maier W, Buller R, Philipp M, Heuser I. The Hamilton Anxiety Scale: reliability, validity and sensitivity to change in anxiety and depressive disorders. J Affect Disord. 1988;14(1):61-8.

12. Rajkumar R. COVID-19 and mental health: A review of the existing literature. Asian J Psychiatr. 2020:52:102066.

13. Pappa S, Ntella V, Giannakas T, Giannakoulis V, Papoutsi E, Katsaounou P. Prevalence of depression, anxiety, and insomnia among healthcare workers during the COVID-19 pandemic: A systematic review and meta-analysis. Brain Behav Immun. 2020 May 8. 